\title{
Devil's Staircase in an Optomechanical Cavity
}

\author{
Hui Wang, Yuvaraj Dhayalan, and Eyal Buks \\ Department of Electrical Engineering, Technion, Haifa 32000 Israel
}

(Dated: October 18, 2018)

\begin{abstract}
We study self-excited oscillation (SEO) in an on-fiber optomechanical cavity. While the phase of SEO randomly diffuses in time when the laser power that is injected into the cavity is kept constant, phase locking may occur when the laser power is periodically modulated in time. We investigate the dependence of phase locking on the amplitude and frequency of the laser power modulation. We find that phase locking can be induced with a relatively low modulation amplitude provided that the ratio between the modulation frequency and the frequency of SEO is tuned close to a rational number of relatively low hierarchy in the Farey tree. To account for the experimental results a one dimensional map, which allows evaluating the time evolution of the phase of SEO, is theoretically derived. By calculating the winding number of the one dimensional map the regions of phase locking can be mapped in the plane of modulation amplitude and modulation frequency. Comparison between the theoretical predictions and the experimental findings yields a partial agreement.

PACS numbers: 46.40.- f, 05.45.- a, 65.40.De, 62.40.+ i
\end{abstract}

Optomechanical cavities [1 7] are widely employed for various sensing [8-11] and photonics [12 -18] applications. Moreover, such systems may allow experimental study of the crossover between classical to quantum realms [3, 1928]. The effect of radiation pressure typically governs the optomechanical coupling (i.e. the coupling between the electromagnetic cavity and the mechanical resonator that serves as a movable mirror) when the finesse of the optical cavity is sufficiently high [3, 5, 23, 29 31], whereas, bolometric effects can contribute to the optomechanical coupling when optical absorption by the vibrating mirror is significant [4, 32 39]. Generally, bolometric effects are dominant in systems comprising of relatively large mirrors, in which the thermal relaxation rate is comparable to the mechanical resonance frequency 37, 38, 40, 41. These systems [4, 32, 34, 40, 42, 43] exhibit many intriguing phenomena such as mode cooling and self-excited oscillation (SEO) [2, 22, 34, 37, 40, 44-47]. It has been recently demonstrated that optomechanical cavities can be fabricated on the tip of an optical fiber [48 58]. These micron-scale devices, which can be optically actuated [59], can be used for sensing physical parameters that affect the mechanical properties of the suspended mirror (e.g. absorbed mass, heating by external radiation, acceleration, etc.).

In a recent study [58] phase locking of SEO has been investigated in an on-fiber optomechanical cavity, which is formed between a fiber Bragg grating (FBG) mirror, serving as a static reflector, and a vibrating mirror, which is fabricated on the tip of a single mode optical fiber. In that experiment [58] SEO [8 11] has been optically induced by injecting a monochromatic laser light into the on-fiber optomechanical cavity. The optically-induced SEO is attributed to the bolometric optomechanical coupling between the optical mode and the mechanical resonator [42, 43]. It was found in [58] that the phase of the SEO can be locked by periodically modulating the laser power that is injected into the cavity. Such phase locking results in entrainment [60 62], i.e. synchroniza- tion 63 65 between the SEO and the external modulation 66, 67]. Synchronization in self-oscillating systems 63, 68 73], in general, can be the result of interaction between systems [74 80], external noise [81 88] or other outside sources, periodic [89 91] or non-periodic [92, 93]. Synchronization can also be activated by applying a delayed feedback 94 97].

In the experiment reported in [58] phase locking [98 103. has been studied for the case where the ratio between the modulation frequency and the frequency of SEO, which is henceforth labeled as $1-\alpha$, was tuned close to two values, the first value $1-\alpha=1$ corresponds to modulation at the SEO frequency, and the second value $1-\alpha=2$ corresponds to modulation at twice the SEO frequency. In the current paper we extend the study and investigate phase locking for arbitrary values of the dimensionless parameter $\alpha$ in the range $[0,1]$. This is done by experimentally mapping the region of phase locking in the plane of the modulation amplitude and modulation frequency. We find that phase locking can be induced with relatively low modulation amplitude provided that $\alpha$ is tuned close to a rational number of relatively low hierarchy in the Farey tree 104]. To account for the experimental results we theoretically evaluate the effect of modulation on the time evolution of the phase of SEO. Some simplifying assumptions and approximations lead to a one dimensional map [Eq. (5) below], which describes the change that is accumulated over a single period of mechanical oscillation in the relative phase between SEO and the modulation, which is labelled by $2 \pi q$. The winding number of the one dimensional map exhibits Devil's staircase (Fig. 2 below) [98, 105 107], i.e. plateaus near rational values of the parameter $\alpha$, corresponding to regions where phase locking [99, 100] occurs. Partial agreement is obtained from the comparison between the experimental findings and theoretical predictions.

The optomechanical cavity, which is schematically shown in Fig. 1(a), was fabricated on the flat polished tip 

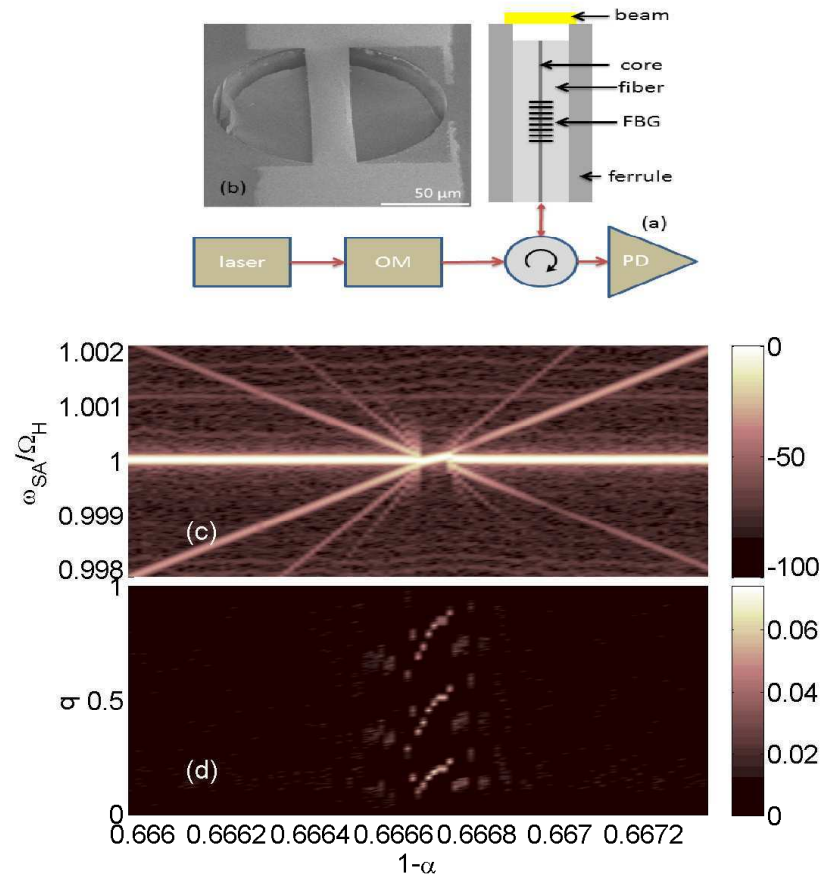

FIG. 1: Experimental setup and phase locking. (a) A schematic drawing of the sample and the experimental setup. An on-fiber optomechanical cavity is excited by a tunable laser with external optical modulator (OM). The mechanical resonator has quality factor $Q=\omega_{0} / 2 \gamma_{0}=3800$ and the cavity has finesse $\beta_{\mathrm{F}}=2.1$. The reflected light intensity is measured using a photodetector (PD), which is connected to both a spectrum analyzer and an oscilloscope (not shown in the sketch). (b) Electron micrograph of the suspended micromechanical mirror, whose mass is $m=1.1 \times 10^{-12} \mathrm{~kg}$. (c) Spectrum analyzer signal in $\mathrm{dB}$ units vs. $1-\alpha$ and normalized measurement frequency $\omega_{\mathrm{SA}} / \Omega_{\mathrm{H}}$. In a region near the point $1-\alpha=2 / 3$ entrainment occurs. The average laser power is set to $12 \mathrm{~mW}$, the wavelength to $\lambda=1545.498 \mathrm{~nm}$ and the dimensionless modulation amplitude to $\beta_{\mathrm{f}}=0.025$. (d) The measured probability distribution $F(q)$ vs. $1-\alpha$. In the same region where entrainment occurs [see panel(c)] the distribution $F(q)$ is peaked near three values [see panel (d)].

of a single mode fused silica optical fiber with outer diameter of $126 \mu \mathrm{m}$ (Corning SMF-28 operating at wavelength band around $1550 \mathrm{~nm}$ ) held in a zirconia ferrule [57]. A 10 - nm-thick chromium layer and a $200 \mathrm{~nm}$ gold layer were successively deposited by thermal evaporation. The bilayer was directly patterned by a focused ion beam to the desired mirror shape $(20-\mu \mathrm{m}$-wide doubly clamped beam). Finally, the mirror was released by etching approximately $12 \mu \mathrm{m}$ of the underlying silica in $7 \% \mathrm{HF}$ acid (90 min etch time at room temperature). The suspended mirror remained supported by the zirconia ferrule, which is resistant to $\mathrm{HF}$.

The static mirror of the optomechanical cavity was provided by a FBG mirror 42] (made using a standard phase mask technique [108], with grating period of
$0.527 \mu \mathrm{m}$ and length $\approx 8 \mathrm{~mm}$ ) having reflectivity band of $0.4 \mathrm{~nm}$ full width at half maximum (FWHM) centered at $1545 \mathrm{~nm}$. The length of the optical cavity was $l \approx 10 \mathrm{~mm}$, providing a free spectral range of $\Delta \lambda=\lambda_{0}^{2} / 2 n_{\text {eff }} l \approx 80$ pm (where $n_{\text {eff }}=1.468$ is the effective refraction index for SMF-28). Monochromatic light was injected into the fiber bearing the cavity on its tip from a laser source with an adjustable output wavelength $\lambda$ and power level $P_{\mathrm{L}}$. The laser was connected through an optical circulator, that allowed the measurement of the reflected light intensity $P_{\mathrm{R}}$ by a fast responding photodetector [see Fig. 1(a)]. The detected signal was analyzed by an oscilloscope and a spectrum analyzer. The experiments were performed in vacuum (at residual pressure below $0.01 \mathrm{~Pa}$ ) at a base temperature of $77 \mathrm{~K}$. The laser power and laser wavelength were first tuned into the regime of SEO before the modulation was turned on.

Phase locking has been measured near all fractions $\alpha=n_{1} / n_{2}$ in the range $0<\alpha<1$, where $n_{2} \in$ $\{1,2,3,4,5\}$. The case $1-\alpha=2 / 3$ is demonstrated by Fig. 1. The plot in panel (c) exhibits the measured signal of a spectrum analyzer, which is connected to the photodetector, vs. normalized modulation frequency $1-\alpha$ and normalized measurement frequency $\omega_{\mathrm{SA}} / \Omega_{\mathrm{H}}$, where $\Omega_{\mathrm{H}} / 2 \pi=236.3 \mathrm{kHz}$ is the frequency of SEO. In the region of phase locking near the point $1-\alpha=2 / 3$ the spectral peak corresponding to SEO coincides with the sideband corresponding to the power modulation. The other spectral lines in panel (c) converging to the central point $1-\alpha=2 / 3$ and $\omega_{\mathrm{SA}} / \Omega_{\mathrm{H}}=1$ represent higher order products of frequency mixing between $\Omega_{\mathrm{H}}$ and $(1-\alpha) \Omega_{\mathrm{H}}$ 109]. The plot in panel (d) of Fig. 1 exhibits the measured probability distribution $F(q)$ of the variable $q$, which represents the relative phase between SEO and the modulation in units of $2 \pi$. The distribution $F(q)$ is extracted from the oscilloscope's data by employing the zero-crossing technique [110]. While $F(q)$ is found to have a nearly uniform distribution away from the point $1-\alpha=2 / 3$, three pronounced peaks are observed in the region of phase locking near that point, suggesting that the relative phase undergoes a limit cycle of period 3 .

To account for the experimental findings we theoretically investigate under what conditions phase locking of SEO is expected to occur. In the limit of small displacement the dynamics of the system can be approximately described using a single evolution equation [43, 111]. The theoretical model that is used to derive the evolution equation [see Eq. (4) below] is briefly described below. Note that some optomechanical effects that were taken into account in the theoretical modeling [43] were found experimentally to have a negligible effect on the dynamics [42] (e.g. the effect of radiation pressure). In what follows such effects are disregarded.

The micromechanical mirror in the optical cavity is treated as a mechanical resonator with a single degree of freedom $x$ having mass $m$ and linear damping rate $\gamma_{0}$ (when it is decoupled from the optical cavity). It is assumed that the angular resonance frequency of the 
mechanical resonator depends on the temperature $T$ of the suspended mirror. For small deviation of $T$ from the base temperature $T_{0}$ (i.e. the temperature of the supporting substrate) it is taken to be given by $\omega_{0}-\beta T_{R}$, where $T_{\mathrm{R}}=T-T_{0}$ and where $\beta$ is a constant. Furthermore, to model the effect of thermal deformation [34] it is assumed that a temperature dependent force given by $m \theta T_{\mathrm{R}}$, where $\theta$ is a constant, acts on the mechanical resonator [39]. When noise is disregarded, the equation of motion governing the dynamics of the mechanical resonator is taken to be given by

$$
\frac{\mathrm{d}^{2} x}{\mathrm{~d} t^{2}}+2 \gamma_{0} \frac{\mathrm{d} x}{\mathrm{~d} t}+\left(\omega_{0}-\beta T_{\mathrm{R}}\right)^{2} x=\theta T_{\mathrm{R}} .
$$

The intra-cavity optical power incident on the suspended mirror is denoted by $P_{\mathrm{L}} I(x)$, where $P_{\mathrm{L}}$ is the injected laser power, and the function $I(x)$ depends on the mechanical displacement $x$ [see Eq. (3) below]. The time evolution of the relative temperature $T_{R}$ is governed by the thermal balance equation

$$
\frac{\mathrm{d} T_{\mathrm{R}}}{\mathrm{d} t}=Q-\kappa T_{\mathrm{R}}
$$

where $Q=\eta P_{\mathrm{L}} I(x)$ is proportional to the heating power, $\eta$ is the heating coefficient due to optical absorption and $\kappa$ is the thermal decay rate.

The function $I(x)$ depends on the properties of the optical cavity that is formed between the suspended mechanical mirror and the on-fiber static reflector. The finesse of the optical cavity is limited by loss mechanisms that give rise to optical energy leaking out of the cavity. The main escape routes are through the on-fiber static reflector, through absorption by the metallic mirror, and through radiation. The corresponding transmission probabilities are respectively denoted by $\mathcal{T}_{\mathrm{B}}, \mathcal{T}_{\mathrm{A}}$ and $\mathcal{T}_{\mathrm{R}}$. In terms of these parameters, the function $I(x)$ is given by [42]

$$
I(x)=\frac{\beta_{\mathrm{F}}\left(1-\frac{\beta_{-}^{2}}{\beta_{+}^{2}}\right) \beta_{+}^{2}}{1-\cos \frac{4 \pi x_{\mathrm{D}}}{\lambda}+\beta_{+}^{2}},
$$

where $x_{\mathrm{D}}=x-x_{\mathrm{R}}$ is the displacement of the mirror relative to a point $x_{\mathrm{R}}$, at which the energy stored in the optical cavity in steady state obtains a local maximum, $\beta_{ \pm}^{2}=\left(\mathcal{T}_{\mathrm{B}} \pm \mathcal{T}_{\mathrm{A}} \pm \mathcal{T}_{\mathrm{R}}\right)^{2} / 8$ and where $\beta_{\mathrm{F}}$ is the cavity finesse. The reflection probability $R_{\mathrm{C}}=P_{\mathrm{R}} / P_{\mathrm{L}}$ is given in steady state by [42, 112] $R_{\mathrm{C}}=1-I(x) / \beta_{\mathrm{F}}$. For sufficiently small $x$, the expansion $I(x)=I_{0}+I_{0}^{\prime} x+$ $(1 / 2) I_{0}^{\prime \prime} x^{2}+O\left(x^{3}\right)$ can be employed, where a prime denotes differentiation with respect to the displacement $x$.

Consider the case where the laser power $P_{\mathrm{L}}$ is modulated in time according to $P_{\mathrm{L}}(t)=P_{0}+P_{1}(t)$, where $P_{0}$ is a constant and $P_{1}(t)$ is assumed to have a vanishing average. When both $P_{1}$ and $I-I_{0}$ are sufficiently small, the problem can be significantly simplified by employing the approximation $Q \simeq \eta P_{0} I+\eta P_{1} I_{0}$. The displacement $x(t)$ is expressed in terms of the complex amplitude $A$ as $x(t)=x_{0}+2 \operatorname{Re} A$, where $x_{0}$, which is given
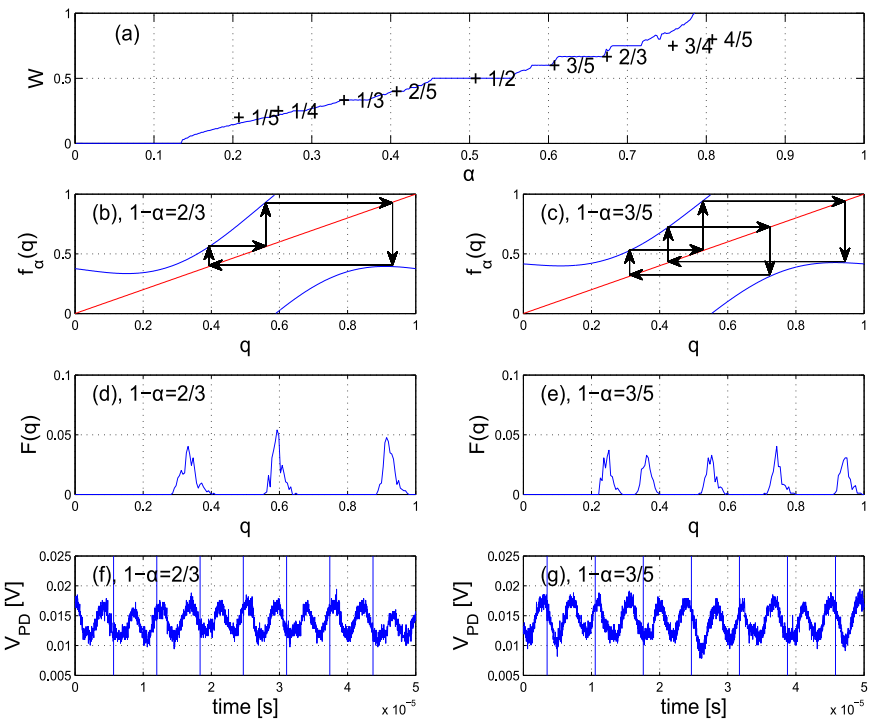

FIG. 2: The winding number and limit cycles. (a) Devil's staircase in the plot of the winding number $W$ vs. $\alpha$ for the case where $\beta_{\mathrm{f}}=0.0355$. Locally stable limit cycles for the case $1-\alpha=2 / 3$ and $\beta_{\mathrm{f}}=0.025$ are presented in panels (b), (d) and (f) and for the case $1-\alpha=3 / 5$ and $\beta_{\mathrm{f}}=0.028$ in panels (c), (e) and (g). The map $f_{\alpha}(q)$ together with the corresponding limit cycle for the case $1-\alpha=2 / 3(1-\alpha=3 / 5)$ is depicted in panel (b) [panel (c)], the experimentally measured probability distribution $F(q)$ in panel (d) [panel (e)], and a sample temporal data in panel (f) [panel (g)] (the vertical lines label the beginning points of each modulation period). In the experimental measurements presented in this plot a pulse power modulation having a rectangular (instead of a sinusoidal) waveform has been employed. For that case the recursive relation $q_{n+1}=f_{\alpha}\left(q_{n}\right)$ is derived by first performing a Fourier decomposition of the rectangular waveform, and then calculating the contribution of each Fourier component using Eq. (6).

by $x_{0}=\eta \theta P_{0} I_{0} / \kappa \omega_{0}^{2}$, is the averaged optically-induced static displacement. For a small displacement, the evolution equation for the complex amplitude $A$ is found to be given by [43, 58]

$$
\dot{A}+\left(\Gamma_{\text {eff }}+i \Omega_{\text {eff }}\right) A=\xi(t)+\vartheta(t),
$$

where both the effective resonance frequency $\Omega_{\text {eff }}$ and the effective damping rate $\Gamma_{\text {eff }}$ are real even functions of $|A|$. To second order in $|A|$ they are given by $\Gamma_{\text {eff }}=\Gamma_{0}+\Gamma_{2}|A|^{2}$ and $\Omega_{\text {eff }}=\Omega_{0}+\Omega_{2}|A|^{2}$, where $\Gamma_{0}=\gamma_{0}+\eta \theta P_{1} I_{0}^{\prime} / 2 \omega_{0}^{2}, \quad \Gamma_{2}=\gamma_{2}+\eta \beta P_{1} I_{0}^{\prime \prime} / 4 \omega_{0}, \gamma_{2}$ is the intrinsic mechanical nonlinear quadratic damping rate [113], $\Omega_{0}=\omega_{0}-\eta \beta P_{1} I_{0} / \kappa$ and $\Omega_{2}=-\eta \beta P_{1} I_{0}^{\prime \prime} / \kappa$. Note that the above expressions for $\Gamma_{\text {eff }}$ and $\Omega_{\text {eff }}$ are obtained by making the following assumptions: $\kappa^{2} / \omega_{0}^{3} \lambda \ll$ $\beta / \theta \ll 1 / 2 \omega_{0} x_{0}$ and $\kappa \ll \omega_{0}$, both typically hold experimentally [42]. The term $\xi(t)$, which is given by $\xi(t)=\Omega_{0}^{-1} \theta T_{\mathrm{R} 1}(t)$, where $T_{\mathrm{R} 1}(t)$ is found by solving Eq. (2) for the case where the the laser power is taken to be $P_{1}(t)$, represents the thermal force that is generated due 


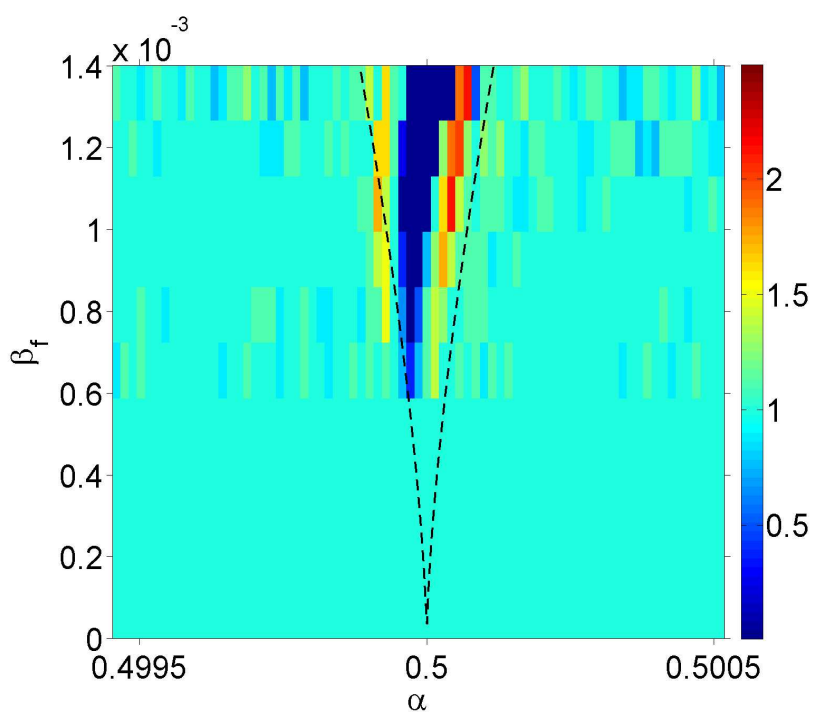

FIG. 3: Arnold tongue near the point $\alpha=1 / 2$. The colormap represents the measured value of the derivative $\mathrm{d} W / \mathrm{d} \alpha$ vs. $\alpha$ and $\beta_{\mathrm{f}}$. The black dotted line represents the theoretically calculated bifurcation line $\beta_{\mathrm{f}}=(81 / 128 \pi)^{1 / 3}(\alpha-1 / 2)^{2 / 3}$. Laser parameters are the same as those listed in the caption of Fig. 1 The experimental value of the dimensionless parameter $\beta_{\mathrm{f}}$ is determined using the following device parameter $\Omega_{\mathrm{H}}^{3} A_{r 0} / \theta \eta I_{0}=0.10 \mathrm{~W}$.

to the power modulation. The fluctuating term [114, 115] $\vartheta(t)=\vartheta_{x}(t)+i \vartheta_{y}(t)$, where both $\vartheta_{x}$ and $\vartheta_{y}$ are real, represents white noise and the following is assumed to hold: $\left\langle\vartheta_{x}(t) \vartheta_{x}\left(t^{\prime}\right)\right\rangle=\left\langle\vartheta_{y}(t) \vartheta_{y}\left(t^{\prime}\right)\right\rangle=2 \Theta \delta\left(t-t^{\prime}\right)$ and $\left\langle\vartheta_{x}(t) \vartheta_{y}\left(t^{\prime}\right)\right\rangle=0$, where $\Theta=\gamma_{0} k_{\mathrm{B}} T_{\text {eff }} / 4 m \omega_{0}^{2}, k_{\mathrm{B}}$ is the Boltzmann's constant and $T_{\text {eff }}$ is the effective noise temperature.

In the absence of laser modulation, i.e. when $P_{1}=0$, the equation of motion (4) describes a van der Pol oscillator [102]. Consider the case where $\Gamma_{2}>0$, for which a supercritical Hopf bifurcation occurs when the linear damping coefficient $\Gamma_{0}$ vanishes. Above threshold, i.e. when $\Gamma_{0}$ becomes negative, the amplitude $A_{r}=|A|$ of SEO is given by $A_{r 0}=\sqrt{-\Gamma_{0} / \Gamma_{2}}$ and the angular frequency $\Omega_{\mathrm{H}}$ of SEO by $\Omega_{\mathrm{H}}=\Omega_{\text {eff }}\left(A_{r 0}\right)$. For our experimental parameters $\left|\Omega_{2}\right| \ll \omega_{0} / A_{r 0}^{2}$, and consequently to a good approximation the dependence of $\Omega_{\mathrm{H}}$ on $A_{r 0}$ can be disregarded.

The laser power modulation $P_{1}(t)$ is taken to be time periodic with angular frequency $(1-\alpha) \Omega_{\mathrm{H}}$, a sinusoidal waveform and an amplitude $P_{\mathrm{p}}$, which is expressed as $P_{\mathrm{p}}=\beta_{\mathrm{f}} \Omega_{\mathrm{H}}^{3} A_{r 0} / \theta \eta I_{0}$, where both $\alpha$ and $\beta_{\mathrm{f}}$ are real dimensionless constants. Let $2 \pi q_{n}$ be the relative phase of SEO with respect to the external modulation after integer number $n$ of periods of mechanical oscillation. Integrating Eq. (4) over a single period of SEO (and disregarding the noise term) yields for the case where $\beta_{\mathrm{f}} \ll 1$ a recursive relation between $q_{n+1}$ and $q_{n}$, which reads

$$
q_{n+1}=f_{\alpha}\left(q_{n}\right)
$$

where

$$
f_{\alpha}(q)=q+\alpha+\frac{2 \beta_{\mathrm{f}} \sin (\pi \alpha) \cos (\pi \alpha+2 \pi q)}{(1-\alpha)\left[1-(1-\alpha)^{2}\right]}
$$

The winding number $W$ is defined by [98]

$$
W=\lim _{n \rightarrow \infty} \frac{q_{n+1}-q_{1}}{n} .
$$

For the case of a limit cycle, the winding number is a rational number given by $W=n_{1} / n_{2}$, where $n_{2}$ is the period of the cycle and $n_{1}$ is the number of sweeps through the unit interval $[0,1]$ in a cycle when the mapping (6]) is considered as modulo 1. Devil's staircase can be seen in Fig. 2(a), in which the winding number $W$ is plotted as a function of $\alpha$ for the case where $\beta_{\mathrm{f}}=0.0355$. Locally stable limit cycles are presented in Fig. 2 for the case $1-\alpha=2 / 3$ and $\beta_{\mathrm{f}}=0.025$ [panels (b), (d) and (f)] and for the case $1-\alpha=3 / 5$ and $\beta_{\mathrm{f}}=0.028$ [panels (c), (e) and $(\mathrm{g})]$. The map $f_{\alpha}(q)$ together with the corresponding limit cycle for the case $1-\alpha=2 / 3(1-\alpha=3 / 5)$ is depicted in panel (b) [panel (c)], the experimentally measured probability distribution $F(q)$ in panel (d) [panel (e)], and a sample temporal data in panel (f) [panel $(\mathrm{g})$ ]. The comparison between the values of $q$ corresponding to the peaks in the measured distribution $F(q)$ [panels (d) and (e)] and the values of $q$ corresponding to the calculated limit cycle [panels (b) and (c)] exhibits a partial agreement.

Regions of phase locking in the plane that is spanned by the modulation parameters (frequency and amplitude) are commonly called Arnold tongues. The Arnold tongue near $\alpha=1 / 2$ is seen in Fig. 3. The colormap exhibits the measured value of the derivative $\mathrm{d} W / \mathrm{d} \alpha$ vs. $\alpha$ and $\beta_{\mathrm{f}}$. Near the point $\alpha=1 / 2$ the Arnold tongue (the region where $\mathrm{d} W / \mathrm{d} \alpha \simeq 0$ in Fig. 3) represents the stability zone of a fixed point of the second order map $\mathcal{F}_{2, \alpha}(q) \equiv f_{\alpha}\left(f_{\alpha}(q)\right)$. To account for the experimental results the behavior of the map $\mathcal{F}_{2, \alpha}(q)$ is theoretically investigated near the point $\alpha=1 / 2$. By expressing $\alpha$ as $\alpha=1 / 2+\epsilon$ and by expanding $\mathcal{F}_{2, \alpha}(q)$ up to first order in $\epsilon$ the fixed point, which is labeled as $q_{\mathrm{f}}$, can be analytically evaluated to lowest nonvanishing order in $\beta_{\mathrm{f}}$. The region of stability, which is found from the requirement that $\left|\mathcal{F}_{2, \alpha}^{\prime}\left(q_{\mathrm{f}}\right)\right|<1$, yields the bifurcation line in the plane spanned by $\alpha$ and $\beta_{\mathrm{f}}$, which is found to be given by $\beta_{\mathrm{f}}=(81 / 128 \pi)^{1 / 3} \epsilon^{2 / 3}$ (see the black dotted line in Fig. 3). The comparison between data and theory yields a moderate agreement.

In summary, Devil's staircase in an on-fiber optomechanical cavity is investigated. The device under study can be employed as a sensor operating in the region of SEO. Future study will address the possibility of reducing phase noise by inducing phase locking in order to enhance sensor's performance. 
This work was supported by the Israel Science Foundation, the bi-national science foundation, the Security Research Foundation in the Technion, the Israel Ministry of Science and the Russell Berrie Nanotechnology Institute.
[1] V. Braginsky and A. Manukin, "Ponderomotive effects of electromagnetic radiation", Soviet Physics JETP, vol. 25, pp. 653, 1967.

[2] K. Hane and K. Suzuki, "Self-excited vibration of a self-supporting thin film caused by laser irradiation", Sensors and Actuators A: Physical, vol. 51, pp. 179$182,1996$.

[3] S. Gigan, H. R. Böhm, M. Paternostro, F. Blaser, J. B. Hertzberg, K. C. Schwab, D. Bauerle, M. Aspelmeyer, and A.Zeilinger, "Self cooling of a micromirror by radiation pressure", Nature, vol. 444, pp. 67-70, 2006.

[4] C. H. Metzger and K.Karrai, "Cavity cooling of a microlever", Nature, vol. 432, pp. 1002-1005, 2004.

[5] T. J. Kippenberg and K. J. Vahala, "Cavity optomechanics: Back-action at the mesoscale", Science, vol. 321, no. 5893, pp. 1172-1176, Aug 2008.

[6] C. Metzger I. Favero, S. Camerer, D. Konig, H. Lorenz, J. P. Kotthaus, and K. Karrai, "Optical cooling of a micromirror of wavelength size", Appl. Phys. Lett., vol. 90, pp. 104101, 2007.

[7] Florian Marquardt and Steven M. Girvin, "Optomechanics", Physics, vol. 2, pp. 40, May 2009.

[8] D. Rugar, H. J. Mamin, and P. Guethner, "Improved fiber-optic interferometer for atomic force microscopy", Applied Physics Letters, vol. 55, no. 25, pp. 2588-2590, 1989.

[9] O. Arcizet, P.-F. Cohadon, T. Briant, M. Pinard, A. Heidmann, J.-M. Mackowski, C. Michel, L. Pinard, O. Français, and L. Rousseau, "High-sensitivity optical monitoring of a micromechanical resonator with a quantum-limited optomechanical sensor", Phys Rev Lett, vol. 97, pp. 133601, Sep 2006.

[10] S. Forstner, S. Prams, J. Knittel, E. D. van Ooijen, J. D. Swaim, G. I. Harris, A. Szorkovszky, W. P. Bowen, and H. Rubinsztein-Dunlop, "Cavity optomechanical magnetometer", Phys. Rev. Lett., vol. 108, pp. 120801, Mar 2012.

[11] S. Stapfner, L. Ost, D. Hunger, J. Reichel, I. Favero, and E. M. Weig, "Cavity-enhanced optical detection of carbon nanotube brownian motion", Applied Physics Letters, vol. 102, no. 15, pp. 151910, 2013.

[12] S. E. Lyshevski and M.A. Lyshevski, "Nano- and microoptoelectromechanical systems and nanoscale active optics", in Third IEEE Conference on Nanotechnology, 2003., Aug 2003, vol. 2, pp. 840-843.

[13] N.A.D. Stokes, F.M.A. Fatah, and S. Venkatesh, "Selfexcited vibrations of optical microresonators", Electronics Letters, vol. 24, no. 13, pp. 777-778, 1988.

[14] M. Hossein-Zadeh and K. J. Vahala, "An optomechanical oscillator on a silicon chip", IEEE J. Sel. Top. Quantum Electron., vol. 16, no. 1, pp. 276-287, Jan 2010.

[15] M. C. Wu, O. Solgaard, and J. E. Ford, "Optical MEMS for lightwave communication", J. Lightwave Technol., vol. 24, no. 12, pp. 4433-4454, Dec 2006.

[16] Matt Eichenfield, Christopher P. Michael, Raviv Perahia, and Oskar Painter, "Actuation of micro- optomechanical systems via cavity-enhanced optical dipole forces", Nature Photonics, vol. 1, pp. 416, 2007.

[17] Gaurav Bahl, John Zehnpfennig, Matthew Tomes, and Tal Carmon, "Stimulated optomechanical excitation of surface acoustic waves in a microdevice", Nature Communications, vol. 2:403, 2011.

[18] NE Flowers-Jacobs, SW Hoch, JC Sankey, A Kashkanova, AM Jayich, C Deutsch, J Reichel, and JGE Harris, "Fiber-cavity-based optomechanical device", Applied Physics Letters, vol. 101, no. 22, pp. 221109, 2012.

[19] JD Thompson, BM Zwickl, AM Jayich, F. Marquardt, SM Girvin, and JGE Harris, "Strong dispersive coupling of a high-finesse cavity to a micromechanical membrane", Nature, vol. 452, no. 7183, pp. 72-75, 2008.

[20] Pierre Meystre, "A short walk through quantum optomechanics", Annalen der Physik, vol. 525, no. 3, pp. 215-233, 2013.

[21] H. J. Kimble, Y. Levin, A. B. Matsko, K. S. Thorne, and S. P. Vyatchanin, "Conversion of conventional gravitational-wave interferometers into quantum nondemolition interferometers by modifying their input and/or output optics", Phys. Rev. D, vol. 65, pp. 022002, Dec 2001.

[22] Tal Carmon, Hossein Rokhsari, Lan Yang, Tobias J. Kippenberg, and Kerry J. Vahala, "Temporal behavior of radiation-pressure-induced vibrations of an optical microcavity phonon mode", Phys. Rev. Lett., vol. 94, pp. 223902, 2005.

[23] O. Arcizet, P. F.Cohadon, T. Briant, M. Pinard, and A. Heidmann, "Radiation-pressure cooling and optomechanical instability of a micromirror", Nature, vol. 444, pp. 71-74, 2006.

[24] A. M. Jayich, J. C. Sankey, B. M. Zwickl, C. Yang, J. D. Thompson, S. M. Girvin, A. A. Clerk, F. Marquardt, and J. G. E. Harris, "Dispersive optomechanics: a membrane inside a cavity", New J. Phys., vol. 10, pp. 095008, Sep 2008.

[25] A. Schliesser, R. Riviere, G. Anetsberger, O. Arcizet, and T. J. Kippenberg, "Resolved-sideband cooling of a micromechanical oscillator", Nat. Phys., vol. 4, pp. 415-419, 2008.

[26] C. Genes, D. Vitali, P. Tombesi, S. Gigan, and M. Aspelmeyer, "Ground-state cooling of a micromechanical oscillator: Comparing cold damping and cavity-assisted cooling schemes", Phys. Rev. A, vol. 77, pp. 033804, Mar 2008.

[27] J. D. Teufel, D. Li, M. S. Allman, K. Cicak, A. J. Sirois, J. D. Whittaker, and R. W. Simmonds, "Circuit cavity electromechanics in the strong coupling regime", arXiv:1011.3067, Nov 2010.

[28] M. Poot and H. S.J. van der Zant, "Mechanical systems in the quantum regime", Phys. Rep., vol. 511, pp. 273335, 2012.

[29] T. J. Kippenberg, H. Rokhsari, T. Carmon, A. Scherer, and K. J. Vahala, "Analysis of radiation-pressure in- 
duced mechanical oscillation of an optical microcavity", Phys. Rev. Lett., vol. 95, pp. 033901, July 2005.

[30] H. Rokhsari, T. Kippenberg, T. Carmon, and K.J. Vahala, "Radiation-pressure-driven micro-mechanical oscillator", Opt. Express, vol. 13, no. 14, pp. 5293-5301, Jul 2005.

[31] Dustin Kleckner and Dirk Bouwmeester, "Sub-kelvin optical cooling of a micromechanical resonator", Nature, vol. 444, pp. 75, 2006.

[32] G. Jourdan, F. Comin, and J. Chevrier, "Mechanical mode dependence of bolometric backaction in an atomic force microscopy microlever", Phys. Rev. Lett., vol. 101, pp. 133904, Sep 2008.

[33] Francesco Marino and Francesco Marin, "Chaotically spiking attractors in suspended-mirror optical cavities", Phys. Rev. E, vol. 83, pp. 015202, Jan 2011.

[34] C. Metzger, M. Ludwig, C. Neuenhahn, A. Ortlieb, I. Favero, K. Karrai, and F. Marquardt, "Self-induced oscillations in an optomechanical system driven by bolometric backaction", Phys. Rev. Lett., vol. 101, pp. 133903, Sep 2008.

[35] J. Restrepo, J. Gabelli, C. Ciuti, and I. Favero, "Classical and quantum theory of photothermal cavity cooling of a mechanical oscillator", Comptes Rendus Physique, vol. 12, pp. 860-870, Nov 2011.

[36] S. D. Liberato, N. Lambert, and F. Nori, "Quantum limit of photothermal cooling", arXiv:1011.6295, Nov 2010.

[37] Florian Marquardt, J. G. E. Harris, and S. M. Girvin, "Dynamical multistability induced by radiation pressure in high-finesse micromechanical optical cavities", Phys. Rev. Lett., vol. 96, pp. 103901, 2006.

[38] M. Paternostro, S. Gigan, M. S. Kim, F. Blaser, H. R. Böhm, and M. Aspelmeyer, "Reconstructing the dynamics of a movable mirror in a detuned optical cavity", New J. Phys., vol. 8, pp. 107, Jun 2006.

[39] D. Yuvaraj, M. B. Kadam, Oleg Shtempluck, and Eyal Buks, "Optomechanical cavity with a buckled mirror", JMEMS, vol. 22, pp. 430, 2013.

[40] K. Aubin, M. Zalalutdinov, T. Alan, R.B. Reichenbach, R. Rand, A. Zehnder, J. Parpia, and H. Craighead, "Limit cycle oscillations in CW laser-driven NEMS", J. MEMS, vol. 13, pp. 1018-1026, 2004.

[41] Simone De Liberato, Neill Lambert, and Franco Nori, "Quantum noise in photothermal cooling", Phys. Rev. A, vol. 83, pp. 033809, Mar 2011.

[42] S. Zaitsev, A. K. Pandey, O. Shtempluck, and E. Buks, "Forced and self-excited oscillations of optomechanical cavity", Phys. Rev. E, vol. 84, pp. 046605, 2011.

[43] S. Zaitsev, O. Gottlieb, and E. Buks, "Nonlinear dynamics of a microelectromechanical mirror in an optical resonance cavity", Nonlinear Dyn., vol. 69, pp. 15891610, 2012.

[44] K. Kim and S. Lee, "Self-oscillation mode induced in an atomic force microscope cantilever", J. Appl. Phys., vol. 91, pp. 4715-4719, 2002.

[45] Thomas Corbitt, David Ottaway, Edith Innerhofer, Jason Pelc, and Nergis Mavalvala, "Measurement of radiation-pressure-induced optomechanical dynamics in a suspended fabry-perot cavity", Phys. Rev. A, vol. 74, pp. 21802, 2006.

[46] Tal Carmon and Kerry J. Vahala, "Modal spectroscopy of optoexcited vibrations of a micron-scale on-chip resonator at greater than 1 ghz frequency", Phys. Rev.
Lett., vol. 98, pp. 123901, 2007.

[47] M. Bagheri, M. Poot, M. Li, W. P. H. Pernice, and H. X. Tang, "Dynamic manipulation of nanomechanical resonators in the high-amplitude regime and non-volatile mechanical memory operation", Nature Nanotechnology, vol. 6, pp. 726-732, 2011.

[48] D. Iannuzzi, S. Deladi, V. J. Gadgil, R. G. P. Sanders, H. Schreuders, and M. C. Elwenspoek, "Monolithic fiber-top sensor for critical environments and standard applications", Applied Physics Letters, vol. 88, no. 5, pp. 053501, 2006.

[49] Cheng Ma and Anbo Wang, "Optical fiber tip acoustic resonator for hydrogen sensing", Opt. Lett., vol. 35, no. 12, pp. 2043-2045, Jun 2010.

[50] D. Chavan, G. Gruca, S. de Man, M. Slaman, J. H. Rector, K. Heeck, and D. Iannuzzi, "Ferrule-top atomic force microscope", Review of Scientific Instruments, vol. 81, no. 12, pp. 123702, 2010.

[51] Khashayar Babaei Gavan, Jan H. Rector, Kier Heeck, Dhwajal Chavan, Grzegorz Gruca, Tjerk H. Oosterkamp, and Davide Iannuzzi, "Top-down approach to fiber-top cantilevers", Opt. Lett., vol. 36, no. 15, pp. 2898-2900, Aug 2011.

[52] Il Woong Jung, B. Park, J. Provine, R.T. Howe, and O. Solgaard, "Highly sensitive monolithic silicon photonic crystal fiber tip sensor for simultaneous measurement of refractive index and temperature", Lightwave Technology, Journal of, vol. 29, no. 9, pp. 1367-1374, 2011.

[53] A. Butsch, M. S. Kang, T. G. Euser, J. R. Koehler, S. Rammler, R. Keding, and P. St.J. Russell, "Optomechanical nonlinearity in dual-nanoweb structure suspended inside capillary fiber", Phys. Rev. Lett., vol. 109, pp. 183904, Nov 2012.

[54] Frank Albri, Jun Li, Robert R J Maier, William N MacPherson, and Duncan P Hand, "Laser machining of sensing components on the end of optical fibres", Journal of Micromechanics and Microengineering, vol. 23, no. 4, pp. 045021, 2013.

[55] AB Shkarin, NE Flowers-Jacobs, SW Hoch, AD Kashkanova, C Deutsch, J Reichel, and JGE Harris, "Optically mediated hybridization between two mechanical modes", Physical review letters, vol. 112, no. 1, pp. 013602, 2014.

[56] Ilya Baskin, D.Yuvaraj, Gil Bachar, Keren Shlomi, Oleg Shtempluck, and Eyal Buks, "Optically induced selfexcited oscillations in an on-fiber optomechanical cavity", JMEMS, vol. 23, pp. 563-569, 2014.

[57] Yuvaraj Dhayalan, Ilya Baskin, Keren Shlomi, and Eyal Buks, "Phase space distribution near the self-excited oscillation threshold", Phys. Rev. Lett., vol. 112, pp. 210403, May 2014.

[58] Keren Shlomi, D Yuvaraj, Ilya Baskin, Oren Suchoi, Roni Winik, and Eyal Buks, "Synchronization in an optomechanical cavity", Physical Review E, vol. 91, no. 3, pp. 032910, 2015.

[59] G. Gruca, D. Chavan, J. Rector, K. Heeck, and D. Iannuzzi, "Demonstration of an optically actuated ferruletop device for pressure and humidity sensing", Sensors and Actuators A: Physical, vol. 190, no. 0, pp. $77-83$, 2013.

[60] Ryan Hamerly and Hideo Mabuchi, "Optical devices based on limit cycles and amplification in semiconductor optical cavities", arXiv:1504.04410, 2015. 
[61] David B Blocher, Alan T Zehnder, and Richard H Rand, "Entrainment of micromechanical limit cycle oscillators in the presence of frequency instability", Microelectromechanical Systems, Journal of, vol. 22, no. 4, pp. 835-845, 2013.

[62] LG Villanueva, E Kenig, RB Karabalin, MH Matheny, Ron Lifshitz, MC Cross, and ML Roukes, "Surpassing fundamental limits of oscillators using nonlinear resonators", Physical review letters, vol. 110, no. 17, pp. 177208, 2013.

[63] Michael Rosenblum and Arkady Pikovsky, "Synchronization: from pendulum clocks to chaotic lasers and chemical oscillators", Contemporary Physics, vol. 44, no. 5, pp. 401-416, 2003.

[64] Christiaan Huygens and Horologium Oscillatorium, "The pendulum clock", Trans RJ Blackwell, The Iowa State University Press, Ames, 1986.

[65] M. C. Cross, A. Zumdieck, Ron Lifshitz, and J. L. Rogers, "Synchronization by nonlinear frequency pulling", Phys. Rev. Lett., vol. 93, pp. 224101, 2004.

[66] Georg Heinrich, Max Ludwig, Jiang Qian, Björn Kubala, and Florian Marquardt, "Collective dynamics in optomechanical arrays", Physical review letters, vol. 107, no. 4, pp. 043603, 2011.

[67] Shreyas Y Shah, Mian Zhang, Richard Rand, and Michal Lipson, "Master-slave locking of optomechanical oscillators over a long distance", Physical review letters, vol. 114, no. 11, pp. 113602, 2015.

[68] Grigory V Osipov, Jürgen Kurths, and Changsong Zhou, Synchronization in oscillatory networks, Springer, 2007.

[69] Arkady Pikovsky, Michael Rosenblum, and Jürgen Kurths, "Phase synchronization in regular and chaotic systems", International Journal of Bifurcation and Chaos, vol. 10, no. 10, pp. 2291-2305, 2000.

[70] Valentin Senderovich Afraimovich, VI Nekorkin, GV Osipov, and AV Gaponov-Grekhov, Stability, structures and chaos in nonlinear synchronization networks, World Scientific, 1994.

[71] Yu I Kuznetsov, PS Landa, AF Ol'Khovoi, and SM Perminov, "Relationship between the amplitude threshold of synchronization and the entropy in stochastic self-excited systems", in Soviet Physics Doklady, 1985, vol. 30, p. 221.

[72] Polina S Landa, Regular and chaotic oscillations, Springer, 2001.

[73] Aleksandr Lvovich Fradkov and Alexander Yu Pogromsky, Introduction to control of oscillations and chaos, vol. 35, World Scientific, 1998.

[74] Louis M Pecora and Thomas L Carroll, "Driving systems with chaotic signals", Physical Review A, vol. 44, no. 4, pp. 2374, 1991.

[75] Louis M Pecora and Thomas L Carroll, "Synchronization in chaotic systems", Physical review letters, vol. 64, no. 8, pp. 821, 1990.

[76] Maria de Sousa Vieira, Allan J Lichtenberg, and Michael A Lieberman, "Synchronization of regular and chaotic systems", Physical Review A, vol. 46, no. 12, pp. R7359, 1992.

[77] Hirokazu Fujisaka and Tomoji Yamada, "Stability theory of synchronized motion in coupled-oscillator systems", Progress of Theoretical Physics, vol. 69, no. 1, pp. 32-47, 1983.

[78] PS Landa and MG Rosenblum, "Synchronization and chaotization of oscillations in coupled self-oscillating systems", Applied Mechanics Reviews, vol. 46, no. 7, pp. 414-426, 1993.

[79] Jerzy Warminski, "Nonlinear normal modes of a selfexcited system driven by parametric and external excitations", Nonlinear dynamics, vol. 61, no. 4, pp. 677689, 2010.

[80] Marc-Antoine Lemonde, Nicolas Didier, and Aashish A Clerk, "Nonlinear interaction effects in a strongly driven optomechanical cavity", Physical review letters, vol. 111, no. 5, pp. 053602, 2013.

[81] A.S Pikovsky, "Synchronization and stochastization of the ensamble of autogenerators by external noise", $R a-$ diophys.Quantum Electron. 27, n.5, 576-581, 1984.

[82] AG Balanov, NB Janson, and PVE McClintock, "Coherence resonance of the noise-induced motion on the way to breakdown of synchronization in chaotic systems", Fluctuation and Noise Letters, vol. 3, no. 02, pp. L113-L120, 2003.

[83] K Czołczynski, P Perlikowski, A Stefański, and T Kapitaniak, "Synchronization of self-excited oscillators suspended on elastic structure", Chaos, Solitons 8 Fractals, vol. 32, no. 3, pp. 937-943, 2007.

[84] AG Balanov, NB Janson, DE Postnov, and Peter VE McClintock, "Coherence resonance versus synchronization in a periodically forced self-sustained system", Physical Review E, vol. 65, no. 4, pp. 041105, 2002.

[85] Xue-Juan Zhang and Guan-Xiang Wang, "Stochastic resonance and signal recovery in two-dimensional arrays of coupled oscillators", Physica A: Statistical Mechanics and its Applications, vol. 345, no. 3, pp. 411-420, 2005.

[86] Michael G Rosenblum, Arkady S Pikovsky, and Jürgen Kurths, "Phase synchronization of chaotic oscillators", Physical Review Letters, vol. 76, no. 11, pp. 1804, 1996.

[87] Arkady S Pikovsky, Michael G Rosenblum, Grigory V Osipov, and Jürgen Kurths, "Phase synchronization of chaotic oscillators by external driving", Physica D: Nonlinear Phenomena, vol. 104, no. 3, pp. 219-238, 1997.

[88] YT Yang, C Callegari, XL Feng, and ML Roukes, "Surface adsorbate fluctuations and noise in nanoelectromechanical systems", Nano letters, vol. 11, no. 4, pp. 17531759, 2011.

[89] AA Koronovskii, MK Kurovskaya, and AE Hramov, "Relationship between phase synchronization of chaotic oscillators and time scale synchronization", Technical physics letters, vol. 31, no. 10, pp. 847-850, 2005.

[90] Alexander P Nikitin and Nigel G Stocks, "Estimation of periodicity in synchronised systems", in Second International Symposium on Fluctuations and Noise. International Society for Optics and Photonics, 2004, pp. 171-181.

[91] Min Qian and Xue-Juan Zhang, "Frequency resonance in stochastic systems", Chinese physics letters, vol. 20, no. 2, pp. 202, 2003.

[92] Seiichiro Nakabayashi and Kohei Uosaki, "Synchronization of electrochemical oscillations with external perturbations", Chemical physics letters, vol. 217, no. 1, pp. 163-166, 1994.

[93] Michael G Rosenblum, Arkady S Pikovsky, Jürgen Kurths, Grigory V Osipov, István Z Kiss, and John L Hudson, "Locking-based frequency measurement and synchronization of chaotic oscillators with complex dynamics", Physical review letters, vol. 89, no. 26, pp. 264102, 2002. 
[94] Natalia B Janson, AG Balanov, and E Schöll, "Delayed feedback as a means of control of noise-induced motion", Physical review letters, vol. 93, no. 1, pp. 010601, 2004.

[95] AG Balanov, Natalia B Janson, and E Schöll, "Control of noise-induced oscillations by delayed feedback", Physica D: Nonlinear Phenomena, vol. 199, no. 1, pp. 1-12, 2004.

[96] E Schöll, AG Balanov, Natalia B Janson, and A Neiman, "Controlling stochastic oscillations close to a hopf bifurcation by time-delayed feedback", Stochastics and Dynamics, vol. 5, no. 02, pp. 281-295, 2005.

[97] Mustapha Hamdi and Mohamed Belhaq, "Quasiperiodic oscillation envelopes and frequency locking in rapidly vibrated nonlinear systems with time delay", Nonlinear Dynamics, vol. 73, no. 1-2, pp. 1-15, 2013.

[98] M. Høgh Jensen, Per Bak, and Tomas Bohr, "Complete devil's staircase, fractal dimension, and universality of mode- locking structure in the circle map", Phys. Rev. Lett., vol. 50, pp. 1637-1639, May 1983.

[99] L.J. Paciorek, "Injection locking of oscillators", Proc IEEE, vol. 53, pp. 1723-1727, 196.

[100] R. Adler, "A study of locking phenomena in oscillators", Proc. IRE, vol. 34, pp. 351-357, 1946.

[101] VS Anishchenko and TE Vadivasova, "Synchronization of self-oscillations and noise-induced oscillations", JOURNAL OF COMMUNICATIONS TECHNOLOGY AND ELECTRONICS C/C OF RADIOTEKHNIKA I ELEKTRONIKA, vol. 47, no. 2, pp. 117-148, 2002.

[102] Manoj Pandey, Richard H Rand, and Alan T Zehnder, "Frequency locking in a forced mathieu-van der polduffing system", Nonlinear Dynamics, vol. 54, no. 1-2, pp. 3-12, 2008.

[103] Serge Dos Santos and Michel Planat, "Generation of 1/f noise in locked systems working in nonlinear mode", $U l$ trasonics, Ferroelectrics and Frequency Control, IEEE Transactions on, vol. 47, no. 5, pp. 1147-1151, 2000.

[104] James Glazier, Albert Libchaber, et al., "Quasiperiodicity and dynamical systems: an experimentalist's view", Circuits and Systems, IEEE Transactions on, vol. 35, no. 7, pp. 790-809, 1988.

[105] E Ben-Jacob, Y Braiman, R Shainsky, and Y Imry, "Microwave-induced devil's staircase structure and chaotic behavior in current-fed josephson junctions", Applied Physics Letters, vol. 38, no. 10, pp. 822-824, 1981.

[106] C Reichhardt and Franco Nori, "Phase locking, devil's staircases, farey trees, and arnold tongues in driven vortex lattices with periodic pinning", Physical review letters, vol. 82, no. 2, pp. 414, 1999.

[107] Seung-Bo Shim, Matthias Imboden, and Pritiraj Mohanty, "Synchronized oscillation in coupled nanomechanical oscillators", Science, vol. 316, no. 5821, pp. 95-99, 2007.

[108] D.Z. Anderson, V. Mizrahi, T. Erdogan, and A.E. White, "Production of in-fibre gratings using a diffractive optical element", Electronics Letters, vol. 29, no. 6, pp. 566-568, 1993.

[109] Thomas Antoni, Kevin Makles, Rémy Braive, Tristan Briant, Pierre-François Cohadon, Isabelle Sagnes, Isabelle Robert-Philip, and Antoine Heidmann, "Nonlinear mechanics with suspended nanomembranes", EPL (Europhysics Letters), vol. 100, no. 6, pp. 68005, 2012.

[110] L. S. Cutler and C. L. Searle, ",some aspects of the theory andmeasurement of frequency fluctuations in frequency standards", ,Proc. IEEE, vol. 54, pp. 136-154, 1966.

[111] Mark Dykman, Xiaolin Chu, and John Ross, "Stationary probability distribution near stable limit cycles far from hopf bifurcation points", Physical Review E, vol. 48, no. 3, pp. 1646, 1993.

[112] Bernard Yurke and Eyal Buks, "Performance of cavityparametric amplifiers, employing kerr nonlinearites, in the presence of two-photon loss", J. Lightwave Tech., vol. 24, pp. 5054-5066, 2006.

[113] S. Zaitsev, O. Shtempluck, E. Buks, and O. Gottlieb, "Nonlinear damping in a micromechanical oscillator", Nonlinear Dynamics, vol. 67, no. 1, pp. 859-883, 2012.

[114] Hannes Risken, The Fokker-Planck Equation: Methods of Solution and Applications, Springer, 1996.

[115] King Yan Fong, Menno Poot, Xu Han, and Hong X Tang, "Phase noise of self-sustained optomechanical oscillators", Physical Review A, vol. 90, no. 2, pp. 023825, 2014. 